\title{
An Analytical Approach for Determining Harmonic Cusps and Torque Dips in Line Start Synchronous Reluctance Motors
}

\author{
Amir Negahdari \\ IEEE, Student Member
}

\author{
Vivek M. Sundaram \\ IEEE, Student Member
}

\author{
Hamid A. Toliyat \\ IEEE, Fellow
}

\author{
Advanced Electric Machines \& Power Electronics (EMPE) Lab \\ Department of Electrical and Computer Engineering \\ Texas A\&M University \\ College Station, Texas 77843-3128
}

\begin{abstract}
Excellence of line start synchronous reluctance motors (LS-SynRM) such as their high efficiency amongst other types of line start motors necessitates the existence of analytical methods in their design. The start-up period of these motors is of high importance and regarding this, the cage design needs to be discussed thoroughly since the squirrel cage is responsible for speeding up the motor to reach at synchronism. One of the issues in this sense is the rich harmonic content of the air-gap MMF arising from irregular bars configuration which afterwards could lead to creation of the torque dips in the torque-speed characteristic of the machine and making it incapable of synchronization under load. An analytical approach to calculate these harmonic effects while also considering the reluctance rotor contribution on them is proposed in this paper. This scheme starts from the stator winding configuration as a known parameter, obtaining the air gap MMF due to only the stator magnetic field, calculating the current and voltages induced through the rotor bars considering the effect of saliency using the model proposed in $[1,2]$. Next, harmonic contribution of the rotor cage in the air gap MMF is derived. Finally, probable harmonic cusps and consequently torque dips is found in the torquespeed characteristic of the motor which is helpful in anticipating the synchronization capability under various loads. The results have been verified with a sample 4-pole, two flux barriers per pole LS-SynRM which suffers crawling due to the existence of the torque dips in its torque-speed characteristic. All simulations have been done by finite element analysis (FEA) software and a comprehensive program was written in Matlab to implement the proposed algorithm.
\end{abstract}

Keywords: Induction motor (IM), Synchronous reluctance motor (SynRM), Line start synchronous reluctance motor (LS-SynRM), Harmonic cusp, Torque dip, Torque-speed characteristic.

\section{INTRODUCTION}

Induction motor (IM) as the most popular line start motor being used for a long time offers some sort of advantages such as ease of use and simple structure, however, suffers low efficiency due to high rotor loss and relative low power factor. These disadvantages persuaded machine designers to start investigating about acceptable alternatives replacing IM in corresponding applications. Primarily, line start permanent magnet motors were proposed and many researches in their design and performance improvement were performed. Even though they were offering significant superiorities over IM such as higher power factor and efficiency, embedding costly permanent magnets in conjunction with the high risk of demagnetization could be mentioned as their deficiencies. Furthermore, their relative complicated manufacturability in comparison with IM did not gain much industrial attraction.

Applications in which higher efficiency is preferred over high power factor, LS-SynRM could be a potential candidate. It should also be added that simple rotor structure, usability of same reference stator of the counterpart IM and being magnet less provides favorability in terms of large scale production. In order to achieve line starting capability in LS-SynRM, a cage needs to be incorporated on the salient rotor. While this cage should take care of accelerating the motor towards synchronous speed, it has to be placed on the rotor such that the saliency of the rotor would not be hurt. Due to the geometrical differences of the rotor in LS-SynRM and IM, cage design in case of LS-SynRM will be more challenging and several issues not existing in IM might be appeared. References [3] and [4] elaborate on various rotor parameters' effects on synchronization capability of LS-SynRM. This paper intends to discuss about a novel issue caused by irregular rotor bars placement inside the reluctance rotor's flux barriers which is called harmonic cusp creation in torquespeed characteristic of LS-SynRM. Cusps and thereafter torque dips could also exist in those IM equipped with improper combination of rotor bars and stator slots. Many detailed discussions about this are given in literature, $[5,6]$. If 
severe torque dips exist in the torque-speed characteristic of the LS-SynRM, it might not be able to synchronize with the grid frequency and starts crawling that is not a desirable mode of operation, [7]. A key point realized and focused in this work is the rotor reluctance effect on harmonic cusps and torque dips of the LS-SynRM. This is proved by using a specific cage configuration both on an IM and an available SynRM, [8]. As it could be seen in Fig. 1, two distinct torque dips at two different slips ( $\sim 1100 \mathrm{rpm}$ in LS-SynRM and $\sim 900$ rpm in IM) exist in IM and LS-SynRM torque-speed characteristic which means presence of saliency on the rotor affects transient behavior of the machine. This further stresses the need for discovery of a method to detect the probable torque dips while also accounting for the rotor reluctance at the same time. An analytical approach to achieve this goal is proposed in this paper in order to predict the probable cusps created after embedding the cage into the reluctance rotor. The theoretical model derived in $[1,2]$ is used to calculate field distribution through the reluctance rotor. In the last section, all of the results obtained by the proposed approach is compared with FEA simulation results to verify the functionality of the proposed method. An available SynRM, [8] is used for simulation purposes. The motor suffers crawling due to the torque dips in its torque-speed characteristic since it has originally been designed as an inverter fed machine and not a grid fed.
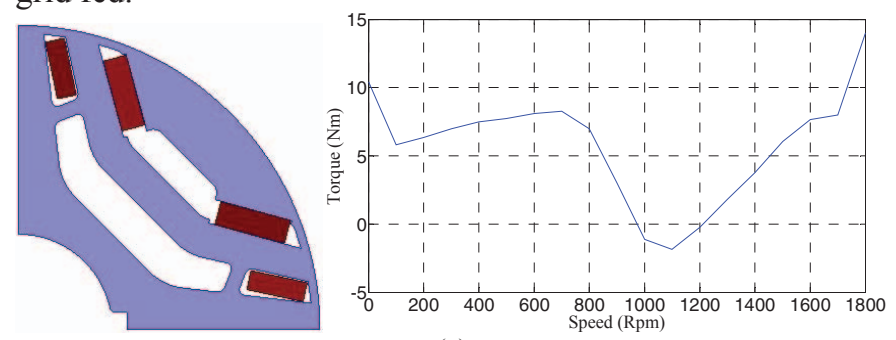

(a)
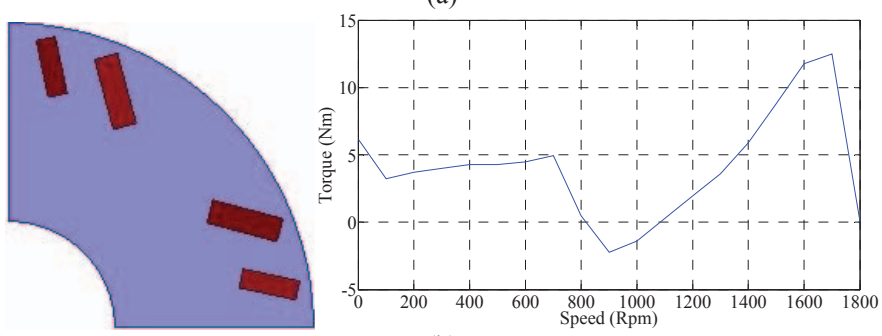

(b)

Fig. 1: Rotor geometry and torque-speed characteristic of: (a) LS-SynRM, and (b) IM with a sample cage configuration.

\section{FIIELD ANALYSIS OVER THE RELUCTANCE ROTOR}

An analytical method for investigation of magnetic field distribution in a reluctance rotor on which several flux barriers might be incorporated is required to be established as a primary step toward harmonic cusps calculation. In here, the well-known analytical approach firstly proposed in [1] and later developed in other papers such as [2] is used. A general reluctance rotor with two hyperbolic-line flux barriers per pole and a stator belonged to a reference NEMA-213 frame IM is considered. Some parameters of IM stator lamination and windings are described in Table 1.
Table. I: IM stator and windings parameters

\begin{tabular}{|c|c|}
\hline Air-gap (inch) & 0.016 \\
\hline Rotor inner diameter (inch) & 1.551 \\
\hline Rotor outer diameter (inch) & 4.968 \\
\hline Stator inner diameter (inch) & 5.00 \\
\hline Stator outer diameter (inch) & 9.00 \\
\hline Number of stator slots & 36 \\
\hline Stator winding coil pitch & 8 \\
\hline Number of windings layer per slot & 2 \\
\hline Number of windings turns per slot & 60 \\
\hline Number of parallel branches per phase & 4 \\
\hline
\end{tabular}

Some simplifying assumptions in this approach which will not be changing the accuracy of the model dramatically are mentioned below:

1 - Stator and rotor yoke materials are assumed to be of infinite permeability so that the magnetic voltage drop across the iron could be neglected. In other words, saturation of permeable material is neglected.

2 - The magnetic flux densities are assumed to be radial in the air-gap and only exists between two adjacent magnetic bridges. Inside the flux barriers, it is perpendicular to both horizontal and radial segments.

3 - A smooth stator and rotor is assumed which means no slot leakage or Carter coefficient is required to be considered.

4 - Air-gap magnetic flux densities only exist between barrier bridges and are zero beneath each bridge.

Based on the aforementioned assumptions and balanced three phase voltages applied to the motor, current through the stator windings can be obtained by solving (1)-(5).

$$
\begin{gathered}
\bar{V}_{s}=R_{s} \bar{i}_{s}+\frac{d \bar{\lambda}_{s}}{d t} \\
j\left(\omega-\omega_{r}\right) \bar{\lambda}_{r}=\bar{R}_{r} \bar{i}_{r}+\frac{d \bar{\lambda}_{r}}{d t} \\
\bar{\lambda}_{s}=L_{l s} \bar{i}_{s}+\bar{L}_{m} \bar{i}_{m} \\
\bar{\lambda}_{r}=\bar{L}_{l r} \bar{i}_{r}+\bar{L}_{m} \bar{i}_{m} \\
\bar{i}_{m}=\bar{i}_{s}+\bar{i}_{r}
\end{gathered}
$$

Where vector and matrix parameters is marked by a "_." on top of them and all equations are written in d-q reference frame. $\mathrm{V}_{\mathrm{s}}$ is the input voltage, $\mathrm{i}_{\mathrm{S}}$ and $\mathrm{i}_{\mathrm{r}}$ are the stator and rotor currents, $i_{m}$ is the magnetizing current, $\lambda s$ and $\lambda_{\mathrm{r}}$ are the stator and rotor flux linkages, $R_{s}$ is the stator winding series resistance, $R_{r}$ and $L_{l r}$ are the rotor resistance and leakage inductance matrices, $\mathrm{L}_{\mathrm{ls}}$ is the stator leakage inductance, $\mathrm{L}_{\mathrm{m}}$ is the magnetizing inductance, $\omega$ is the grid frequency and $\omega_{\mathrm{r}}$ is the rotor mechanical speed. Now, the stator MMF in rotating reference frame can be calculated as: 


$$
\begin{aligned}
& F_{d}=\frac{4}{\pi} \frac{m / 2\left(\sqrt{2} I_{d}\right) N k_{w}}{P} \cos \frac{P}{2} \theta \\
& F_{q}=\frac{4}{\pi} \frac{m / 2\left(\sqrt{2} I_{q}\right) N k_{w}}{P} \sin \frac{P}{2} \theta
\end{aligned}
$$

$F_{d}, F_{q}, I_{d}, I_{q}$ are $d$ and $q$ components of stator MMF and currents, ' $\mathrm{m}$ ' is the number of phases, ' $\mathrm{P}$ ' is the number of poles, ' $\mathrm{N}$ ' is the number of turns per phase and $\mathrm{K}_{\mathrm{w}}$ is winding factor.

A general geometry of SynRM is shown in Fig. 2 in conjunction with different dimensions and fields through it. Two general closed paths $a b c$ and $d e f g$ is considered and Ampere's law is written along them. $a b c$ is representative of those paths crossing radial and horizontal edges of barriers inside the rotor only and defg represents those paths cutting the horizontal edge and air gap as well as containing the stator.

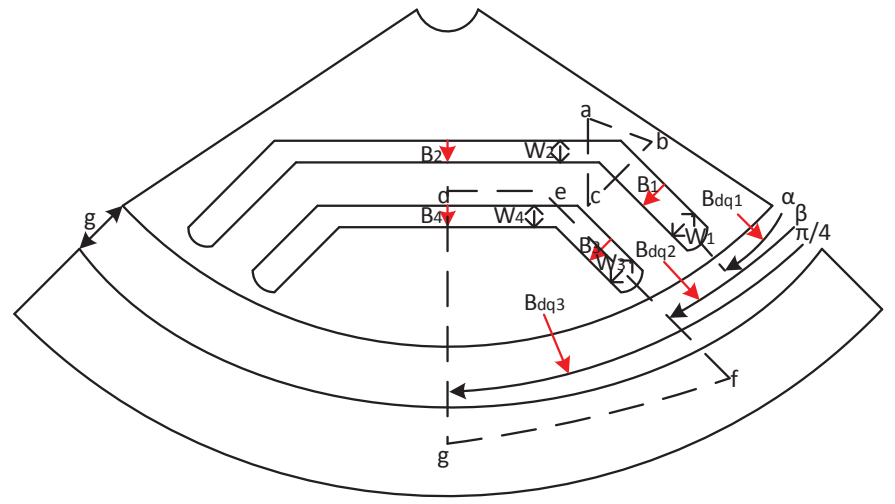

Fig. 2: General SynRM rotor geometry and flux density distribution.

Based on assumption 1, no magnetic voltage drop exists through the core and only air-gap and flux barriers' magnetic fields are accounted. Path $a b c$ results in (8) and (9) and path defg results in (10), (11) and (12). Following equations represent the q-axis fields:

$$
\begin{array}{cc}
B_{1} W_{1}-B_{2} W_{2}=0 & \\
B_{3} W_{3}-B_{4} W_{4}=0 & 0<\theta<\alpha \\
B_{q 1} g=F_{q}(\theta) & \alpha<\theta<\beta \\
B_{q 2} g+B_{2} W_{2}=F_{q}(\theta) & \beta<\theta<\frac{\pi}{4} \\
B_{q 3} g+B_{4} W_{4}=F_{q}(\theta) &
\end{array}
$$

$\mathrm{B}_{\mathrm{q} 1}, \mathrm{~B}_{\mathrm{q} 2}, \mathrm{~B}_{\mathrm{q} 3}, \mathrm{~B}_{1}, \mathrm{~B}_{2}, \mathrm{~B}_{3}, \mathrm{~B}_{4}$ form seven unknowns whereas 5 equations are derived. The two other equations will be obtained by applying the law of consistency of flux in a lossless material. Again according to first assumption, flux entering any segment of the rotor should equal flux leaving it. Considering the segments beneath the bottom barrier and between two barriers shown in blue and green in Fig. 3 and equating fluxes exiting the regions to those entering the regions, (13) and (14) will be derived:

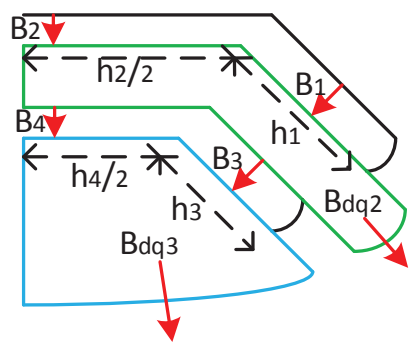

Fig. 3: Cutouts of rotor iron sections. (Blue: beneath the bottom barrier and green: between two barriers)

$$
\begin{gathered}
\frac{D}{2} \int_{\beta}^{\frac{\pi}{4}} B_{q 3} d \theta=B_{3} h_{3}+B_{4} \frac{h_{4}}{2} \\
\frac{D}{2} \int_{\alpha}^{\beta} B_{q 2} d \theta+B_{3} h_{3}+B_{4} \frac{h_{4}}{2}=B_{1} h_{1}+B_{2} \frac{h_{2}}{2}
\end{gathered}
$$

$h_{1}, h_{2}, h_{3}$ and $h_{4}$ are the length of barriers corresponding to $W_{1}$, $\mathrm{W}_{2}, \mathrm{~W}_{3}$ and $\mathrm{W}_{4}$. The same relations remain valid for d-axis flux densities considering no d-axis flux flowing through the barriers:

$$
\begin{aligned}
B_{d 1} g & =F_{d}(\theta) & & 0<\theta<\alpha \\
B_{d 2} g & =F_{d}(\theta) & & \alpha<\theta<\beta \\
B_{d 3} g & =F_{d}(\theta) & & \beta<\theta<\frac{\pi}{P}
\end{aligned}
$$

In this case, $(15,16$ and 17) can be solved independently and no additional equation is required.

\section{CAGE EQUiVAlENT CIRCUIT INCORPORATION}

Using the procedure described in previous section, $\mathrm{d}$ and $\mathrm{q}$ axes magnetic flux densities along the air-gap and flux barriers could be obtained. In this section, an aluminum cage placement inside the flux barriers is studied. By placing the cage inside the barriers, asynchronous torque required in startup can be achieved whereas saliency of the rotor is not suffered at the same time. It is assumed that the cage is only placed inside the radial portions of barriers, however, this is not a major assumption since those bars closer to the periphery of the rotor are more effective in torque production than those which are more towards the center. Cage bars are shown in solid brown in Fig. 4. Each two adjacent bars are treated as a winding. This is valid since bars are short circuited via an endring and the current flows inward from one bar and returns outward from the adjacent one so each bar is similar to a single turn coil and two bars form a winding. Moreover, most of the d-axis flux flows in the actual d-axis and not through the barriers whereas almost all of q-axis flux flows through the barriers. Therefore, instead of $\mathrm{B}_{\mathrm{q} 1}$ in section II, $\mathrm{B}_{\mathrm{d} 1}$ is only considered and $\mathrm{B}_{\mathrm{d} 2}$ and $\mathrm{B}_{\mathrm{d} 3}$ are neglected as shown in Fig. 4. Based on these assumptions, flux linking each winding could be calculated as shown below: 


$$
\begin{aligned}
& \phi_{1}=2\left(\frac{D}{2}\right) l\left\{\int_{0}^{\alpha} B_{d 1}(\theta) d \theta\right\} \\
& \phi_{2}=\left(\frac{D}{2}\right) l\left\{\int_{\alpha}^{\beta} B_{q 2}(\theta) d \theta\right\} \\
& \phi_{3}=2\left(\frac{D}{2}\right) l\left\{\int_{\beta}^{\frac{\pi}{P}} B_{q 3}(\theta) d \theta\right\}
\end{aligned}
$$

$\phi_{1}, \phi_{2}, \phi_{3}$ are the fluxes linking the circuit comprising bars 1 and 4 of the adjacent pole, bars 1 and 2, and bars 2 and 3 respectively, ' 1 ' is the active motor stack length and ' $\mathrm{D}$ ' is the rotor outer diameter. Basically, by integration of the calculated flux densities which are functions of spatial angle and time over the periphery of the rotor, the flux linkages are obtained.

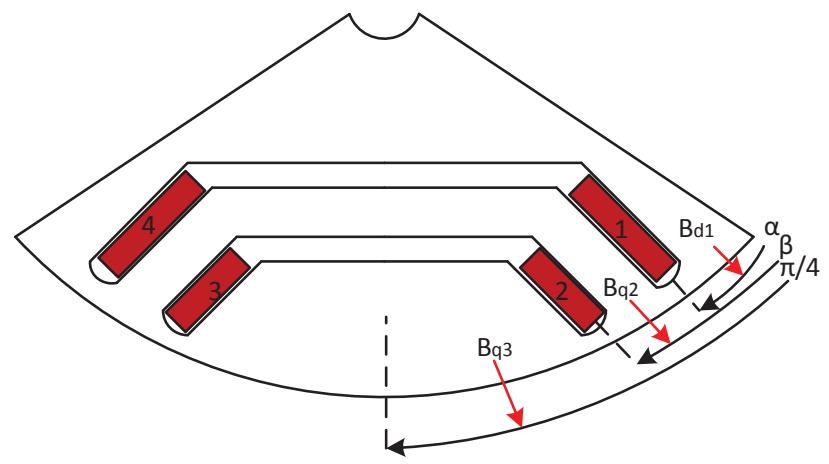

Fig. 4: General LS-SynRM rotor geometry.

Any time varying flux linkage induces voltage across the conductor it is linking. Calculated fluxes need to be derivated with repect to time in order to derive the voltages induced across each branch of end-ring:

$$
\begin{aligned}
& v_{e 1}=\frac{d \phi_{1}}{d t} \\
& v_{e 2}=\frac{d \phi_{2}}{d t} \\
& v_{e 3}=\frac{d \phi_{3}}{d t}
\end{aligned}
$$

$\mathrm{V}_{\mathrm{e} 1}, \mathrm{~V}_{\mathrm{e} 2}, \mathrm{~V}_{\mathrm{e} 3}$ are the voltages induced across the end-ring branches connecting bars 1 and 4 of the adjacent pole, bars 1 and 2 , and bars 2 and 3 respectively.

By knowing the impedances of end-ring branches, current flowing through each end-ring segment can be calculated:

$$
\begin{aligned}
& Z_{e 1}=R_{e 1}+j \omega L_{e 1} \\
& Z_{e 2}=R_{e 2}+j \omega L_{e 2} \\
& Z_{e 3}=R_{e 3}+j \omega L_{e 3}
\end{aligned}
$$

$Z_{e}, R_{e}, L_{e}$ are impedance, resistance and inductance of end-ring branches. $\omega$ also stands for the corresponding angular frequency of the rotor. Presence of the rotor angular frequency in the above equations highlights the important point of rotor slip impact on the cage current harmonic spectrum and hence different harmonic currents both in terms of order and magnitude might flow through the end-ring segments and bars:

$$
\begin{aligned}
& i_{e 1}=\frac{v_{e 1}}{Z_{e 1}} \\
& i_{e 2}=\frac{v_{e 2}}{Z_{e 2}} \\
& i_{e 3}=\frac{v_{e 3}}{Z_{e 3}}
\end{aligned}
$$

$\mathrm{i}_{\mathrm{e}}$ is the end-ring segment current.

Despite the IM, the end-ring impedances are not equal in this case and modifications are required to account for unequality in end-ring segments. This phenomenon will obviously be seen later in the result section. Fig. 5 shows the cage equivalent circuit including bars and end-rings for the LS-SynRM. Applying KVL and KCL in the circuitral network of any arbitrary LS-SynRM with end-ring currents as known parameters, all of the currents and voltages of the circuit can be found.

Currents flowing through the bars will contribute on air-gap MMF and flux density. For instance, air-gap MMF generated by bars 1, 2 and 3 shown in one pole in Fig. 4 and bar 16 from adjacent pole can be written as:

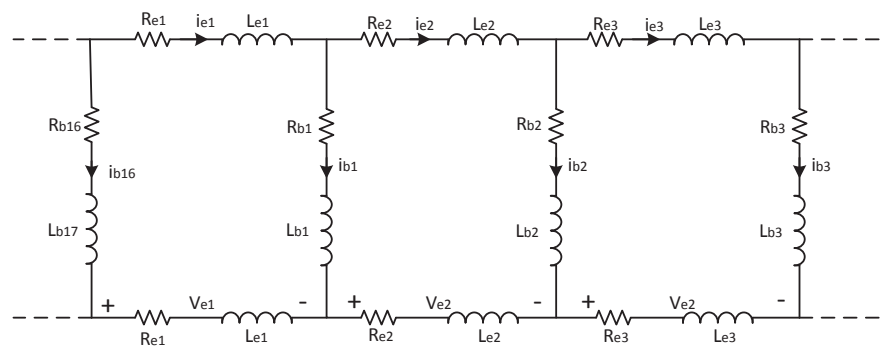

Fig. 5: Cage equivalent circuit per pole.

$$
\begin{gathered}
M M F_{b 1}=\frac{4}{\pi} \frac{i_{b 1}}{2} \sin \frac{P(\theta-\alpha)}{2} \\
M M F_{b 2}=\frac{4}{\pi} \frac{i_{b 2}}{2} \sin \frac{P(\theta-\beta)}{2} \\
M M F_{b 3}=\frac{4}{\pi} \frac{i_{b 3}}{2} \sin \frac{P(\theta-\pi / 2+\alpha+\beta)}{2} \\
M M F_{b 16}=\frac{4}{\pi} \frac{i_{b 16}}{2} \sin \frac{P(\theta+\alpha)}{2}
\end{gathered}
$$


$\mathrm{MMF}_{\mathrm{b}}$ is the MMF generated by each bar, $\mathrm{i}_{\mathrm{b}}$ is the corresponding bar's current and $\theta$ is the variable representing angular displacement with reference point at the beginning of shown pole. The net air-gap MMF created by the rotor is the superposition of all bars' MMFs. It should be noted that calculation of bars' currents in one pole is sufficient and those in other poles could be derived by only phase shifting with regards to their corresponding bars in the refernce pole.

$$
\begin{gathered}
M M F_{p 1}=\sum_{n=1}^{4}\left(\frac{4}{\pi} \frac{i_{b n}}{2} \sin \frac{P\left(\theta-\theta_{n}\right)}{2}\right) \\
M M F_{p 2}=e^{j \pi / 2} \sum_{n=1}^{4}\left(\frac{4}{\pi} \frac{i_{b n}}{2} \sin \frac{P\left(\theta-\left(\theta_{n}+\frac{\pi}{2}\right)\right)}{2}\right) \\
M M F_{p 3}=e^{j \pi} \sum_{n=1}^{4}\left(\frac{4}{\pi} \frac{i_{b n}}{2} \sin \frac{P\left(\theta-\left(\theta_{n}+\pi\right)\right)}{2}\right) \\
M M F_{p 4}=e^{j 3 \pi / 2} \sum_{n=1}^{4}\left(\frac{4}{\pi} \frac{i_{b n}}{2} \sin \frac{P\left(\theta-\left(\theta_{n}+\frac{3 \pi}{2}\right)\right)}{2}\right)
\end{gathered}
$$

Pole in this approach is meant bars $1,2,3$ and 16 which is the adjacent bar to 1 belonged to previous rotor pole. $\mathrm{MMF}_{\mathrm{p}}$ is the air-gap MMF produced by bars of the corresponding pole and the total rotor MMF will be:

$$
M M F_{\text {rotor }}=\sum_{n=1}^{4} M M F_{p n}
$$

By determining the rotor air gap MMF, the air gap magnetic flux density and its spatial harmonics required for calculation of parasitic torques, could also be derived by using appropriate closed paths to write Ampere's law. The counterpart air gap magnetic flux density when no cage is inserted on the rotor and only stator is excited was already obtained as well. Based on [6] and [7], it is the interaction of stator and rotor air gap flux densities that creates asynchronous torques. Magnitude and phase angles of different harmonic orders available in the rotor and stator air gap flux density affect this torque as expressed in (39).

$$
T_{\text {asyn }}=\frac{P D \pi \lg \lambda B_{\mu} B_{\lambda}}{2 \mu_{0}} \cos \left(\psi_{\lambda} \pm \psi_{\mu}\right)
$$

' $\mathrm{P}$ ' stands for the number of poles, ' $\mathrm{D}$ ' for the rotor outer diameter, ' 1 ' for the stack length, $\mu_{0}$ for permeability of free space, ' $g$ ' for the air gap length, $\lambda$ and $\mu$ for the ordinal number of stator and rotor magnetic flux dendity harmonics, $\mathrm{B}_{\lambda}$ and $\mathrm{B}_{\mu}$ for their corresponding magnitude and $\Psi_{\lambda}$ and $\Psi_{\mu}$ for their phase angles, respectively. Considering the torque production mechanism in IM, if a stator MMF harmonic with the same ordinal number to that of the rotor exists, these two components of air gap MMFs are capable of producing asynchronous parasitic torques based on (39).

\section{RESULTS}

The applicability and performance of this approach was verified by means of the LS-SynRM shown in Fig (1-a). An existing SynRM was chosen and the shown sample cage configuration was embedded on its rotor. The resulting torquespeed characteristic proves a large dip at around $1100 \mathrm{rpm}$ which has risen due to rich air gap MMF harmonic content. The most significant harmonics of airgap MMF created by the rotor is shown in Fig. 6. The magnitudes have been normalized with respect to the fundamental harmonic and explained in per unit to convey a better insight. The rotor slip directly influences the air gap MMF harmonic spectrum. Hence, the results are reported in three different sample slips i.e., $0.33,0.55$ and 0.77 .

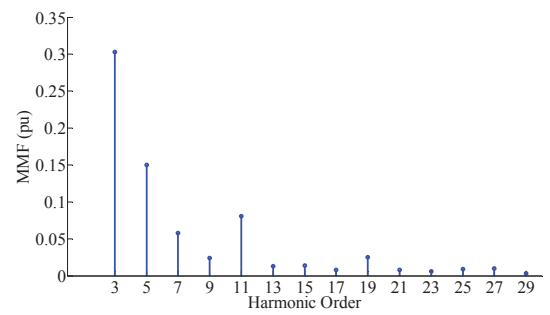

(a)

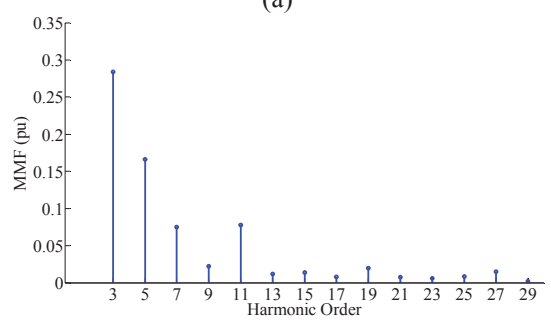

(b)

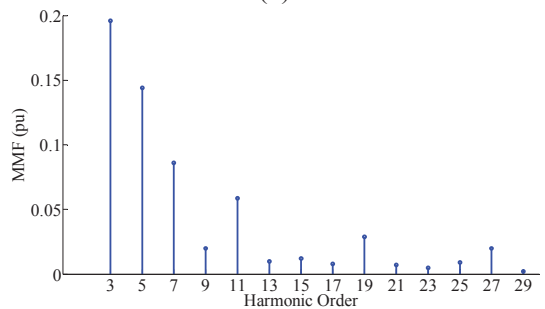

(c)

Fig. 6: Harmonic content of air gap MMF created by the rotor at: (a) 1200 rpm, (b) $800 \mathrm{rpm}$, and (c) $400 \mathrm{rpm}$.

Each of these available harmonics will create its own associated torque-speed characteristic. Fig. (7-a) shows these curves for the first four significant harmonics represented in Fig. 6. The superposition of these harmonics is plotted in Fig. (7-b) representing an approximation of total torque-speed characteristic of the LS-SynRM.

The general trend of the LS-SynRM torque-speed curve is emulated by the proposed method. The differences in torque values are expectable due to several simplifying assumptions made while developing the theory. Furthermore, the analytical curve is plotted only for some dominant harmonics and all the other higher order harmonics are neglected. 


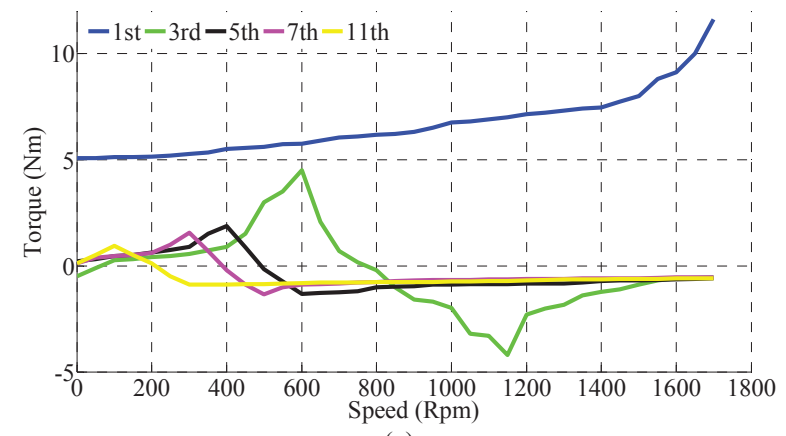

(a)

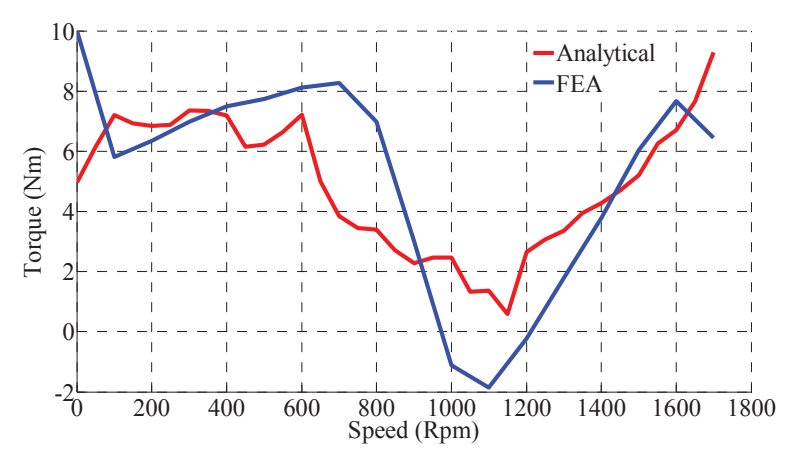

(b)

Fig. 7: (a) Torque-speed curve of significant harmonics, and (b) Total torquespeed curve of the machine vs simulation result.

The obtained result satisfies the desired goal which was detection of those torque dips which are avoiding the motor from speeding up further. In this example, the dip around 1100 rpm is causing difficulty at start-up and this motor is actually trapped around the same speed. The rated load of this motor should be around $11.2 \mathrm{Nm}$ at $1100 \mathrm{rpm}$ and the proposed approach predicts $0.6 \mathrm{Nm}$ developed electromagnetic torque. The less accuracy at lower speeds is because of higher harmonics contribution at lower speeds as could be seen in Fig. (6-c). Since bigger dips are mainly due to lower order harmonics happening at higher speeds and these harmonics usually are significant and easier to detect, it could be deduced that synchronization capability of LS-SynRM could be acceptably anticipated with the proposed algorithm. Moreover, dips at lower speeds are fairly small and will not prevent the motor of accelerating specially in case of LS-SynRM that usually are connected to fan and pump loads with low starting torques.

\section{CONCLUSION}

An analytical solution for detection of torque dips in torquespeed characteristic of LS-SynRM was described. The risk of unsuccessful synchronization because of irregular cage configuration and lack of any theoretical approach in LSSynRM design were the strong motivations behind this work. The proposed model in $[1,2]$ was used to consider the effect of rotor reluctance. Results obtained from this model were combined into the cage. The cage mounted on the rotor was converted to several single turn windings and currents of rotor bars were calculated. Afterwards, parasitic torques due to the air gap flux density harmonics were calculated based on rotor and stator air gap MMF. The most important finding of this research was the rotor reluctance contribution on creation of new harmonic cusps which was considered in this approach. The same task had been already done for pure IM with symmetric cage in $[5,6]$. As the final testimony, this approach could be extended to any number of poles and flux barriers, however, four pole and two flux barriers per pole was only considered in this paper. As future work, some modifications can be applied to avoid the stated simplifying assumptions such as neglecting saturation which ultimately will lead to increase in accuracy of the results.

\section{ACKNOWLEGMENT}

The authors would like to thank ANSYS for their support of finite element analysis software.

\section{References}

[1] V. B. Honsinger, "The Inductances Ld and Lq of Reluctance Machines," in IEEE Transactions on Power Apparatus and Systems, vol. PAS-90, No. 1, pp. 298-304, Jan. 1971.

[2] B. Nikbakhtian, S. Talebi, P. Niazi, and H.A. Toliyat, "An analytical model for an N-flux barrier per pole permanent magnet-assisted synchronous reluctance motor," in Electric Machines and Drives Conference, 2009. IEMDC '09. IEEE International, pp.129-136, 3-6 May 2009.

[3] M. Gamba, G. Pellegrino, A. Vagati, and F. Villata, "Design of a linestart synchronous reluctance motor," in Electric Machines \& Drives Conference (IEMDC), 2013 IEEE International, pp.648-655, 12-15 May, 2013.

[4] M. Gamba, E. Armando, G. Pellegrino, A. Vagati, B. Janjic, and J. Schaab, "Line-start synchronous reluctance motors: Design guidelines and testing via active inertia emulation," in Energy Conversion Congress and Exposition (ECCE), 2015 IEEE, pp.4820-4827, 20-24 Sept. 2015.

[5] H.A. Toliyat and G.B. Kliman, "Induction Motor Analysis and Design," Handbook of Electric Motors, $2^{\text {nd }}$ ed, Boca Raton, FL: CRC Press, 2004.

[6] B. Heller and V. Hamata, "Torques," Harmonic Field Effects in Induction Machines, Amsterdam, Netherlands: Elsevier Scientific Publishing Company, 1977.

[7] A. Negahdari, and H. A. Toliyat, "Studying Crawling Effect in LineStart Synchronous Reluctance Motors (LS-SynRM)," presented at International Symposium on Industrial Electronics (ISIE), Santa Clara, California, June 2016.

[8] R. Vartanian, and H.A. Toliyat, "Design and comparison of an optimized permanent magnet assisted synchronous reluctance motor (PMa-SynRM) with an induction motor with identical NEMA frame stators, "in Electric Ship Technologies Symposium, ESTS 2009. IEEE, pp.107-112, 20-22 April, 2009.

[9] S. Talebi, P. Niazi, and H.A. Toliyat, "Design of Permanent MagnetAssisted Synchronous Reluctance Motors Made Easy," Proceedings of the 2017 IEEE Industry Applications Conference, Annual Meeting, pp.2242-2248, 23-27 Sept. 2007.

[10] A. Castagnini, T. Känsäkangas, J. Kolehmainen and P. S. Termini, "Analysis of the starting transient of a synchronous reluctance motor for direct-on-line applications," 2015 IEEE International Electric Machines \& Drives Conference (IEMDC), Coeur d'Alene, ID, 2015, pp. 121-126. 